\title{
AVALIAÇÃO DA RESISTÊNCIA AO DESGASTE DE TRÊS RESINAS PARA COROAS TEMPORÁRIAS APÓS SIMULAÇÃO DE UM ANO DE ESCOVAÇÃO
}

\section{EVALUATION OF THE WEAR RESISTANCE OF THREE RESINS FOR TEMPORARY CROWNS AFTER SIMULATION OF ONE YEAR OF TOOTHBRUSHING}

\author{
Renato Sussumu Nishioka* \\ Alfredo Mikail Melo Mesquita ** \\ Alberto Noriyuki Kojima *** \\ Gilberto Duarte Filho ***** \\ Luis Gustavo Vasconcelos *****
}

\begin{abstract}
RESUMO
Introdução: O propósito deste estudo é comparar a resistência ao desgaste de três resinas para confecção de coroas temporárias (InstaTemp Provisional Crown \& Bridge - Sterngold ImplaMed; Perfect Temp Discus Dental; Duralay - Reliance Dental), após a simulação de um ano de escovação. Métodos: Foram confeccionados 12 corpos-de-prova $(\mathrm{n}=12)$ com $5 \mathrm{~mm}$ de diâmetro por $3 \mathrm{~mm}$ de espessura, de cada marca comercial, obedecendo às especificações do fabricante. Foi utilizada uma matriz, usinada em aço inoxidável, contendo três orifícios para que fossem assentados os corpos-de-prova das diferentes marcas, sendo que apenas $1 \mathrm{~mm}$ do corpo de prova ficou saliente em relação à superfície da base de aço. Os corpos-de-prova foram acondicionados em água destilada, por catorze dias, a uma temperatura de $37^{\circ} \mathrm{C}$, sendo que todas as amostras foram pesadas em uma balança analítica Mettler Toledo, modelo AB204. A simulação da resistência ao desgaste foi realizada em um simulador de escovação no Centro de Pesquisa e Tecnologia da Johnson e Johnson. As escovas foram trocadas a cada noventa minutos de simulação, e após o total de seis horas, o que corresponderia a um ano de escovaçáo, os corpos-de-prova foram pesados novamente na mesma balança analítica. Resultados e Conclusão: Os resultados obtidos (antes - depois) foram submetidos aos testes t-student e Tukey, ao nível de significância de 5\%. Verificou-se que a resina Insta Temp foi a mais resistente ao desgaste, devido à perda de peso sempre ter sido menor que a de Perfect Temp e Duralay.
\end{abstract}

DESCRITORES: Escovação dentária - Resinas

\section{ABSTRACT}

Introduction: This study compares the wear resistance of three resins for temporary crowns (InstaTemp Provisional Crown \& Bridge - Sterngold ImplaMed; Perfect Temp - Discus Dental; Duralay - Reliance Dental), after one year of toothbrushing simulation. Methods: For this purpose twelve specimens were made with $5 \mathrm{~mm}$ of diameter by $3 \mathrm{~mm}$ of thickness of each commercial trend, following the manufacturer's specifications. A base of stainless steel containing three holes was made so that the specimens of the different manufactures were placed with $1 \mathrm{~mm}$ above the flat surface of the stainless steel base. The samples were conditioned in distilled water, for fourteen days to a temperature of $37^{\circ} \mathrm{C}$. The samples were weight initially in an analytical scale - Mettler Toledo, model AB204. The simulation of the wear resistance was accomplished in a toothbrushing simulator in the Center of Research and Technology of Johnson and Johnson, the brushes were changed every ninety minutes of simulation, for a period of six hours, which corresponds to one year of toothbrushing. Results and conclusion: The specimens were weight again in the same analytical scale. The results obtained were submitted to the t-Student and Tukey test, in the meaning of $5 \%$. It was conclued that the InstaTemp resin proved to be more wear resistant when compared to Duralay and PerfecTemp. It showed a less of weight always smaller than the others.

DESCRIPTORS: Tootbrushing - Resins

* Prof. Dr. da Disciplina de Prótese Parcial Fixa do Departamento de Materiais Odontológicos e Prótese da Faculdade de Odontologia de São José dos Campos - Universidade do Estado de São Paulo - UNESP

** Doutarando em Odontologia Restauradora, Especialidade Prótese Dentária pela Faculdade de Odontologia de São José dos Campos - Universidade do Estado de São Paulo - UNESP

*** Doutarando em Odontologia Restauradora, Especialidade Prótese Dentária pela Faculdade de Odontologia de São José dos Campos - Universidade do Estado de São Paulo - UNESP

**** Mestre em Odontologia Restauradora, Especialidade Prótese Dentária pela Faculdade de Odontologia de São José dos Campos - Universidade do Estado de São Paulo - UNESP

***** Especialista em Prótese Dentária pela Associação dos ex-alunos (AEXAFO) - Faculdade de Odontologia de São José dos Campos - Universidade do Estado de São Paulo - UNESP 
Nishioka RS, Mesquita AMM, Kojima AN, Duarte Filho G, Vasconcelos LG. Avaliação da resistência ao desgaste de três resinas para coroas temporárias após simulação de um ano de escovação. Revista de Odontologia da Universidade Cidade de São Paulo 2008 janabr; 20(1):46-51

\section{INTRODUÇÃO}

A coroa temporária deveria ser realizada de maneira a ser considerada como o protótipo da restauração definitiva, (Nishioka e Bottino ${ }^{22}$ 1989), para reabilitar o paciente estética e funcionalmente, promover proteção pulpar, saúde periodontal, manter o dente em posição no arco, resistir aos esforços mastigatórios, permitir correta higienização e reembasamentos, (Bargui e Simmons ${ }^{3}$ 1976). Objetivando a saúde periodontal, as superfícies das restauraçóes temporárias deveriam ter adequado polimento para minimizar o acúmulo de biofilme, além disso seria importante que houvesse integridade marginal, pois esses fatores freqüentemente são a causa de inflamação gengival (Carranza ${ }^{4}$ 1992).

Por isso, o estudo da perda de massa resultante do contato entre dois corpos, que pode ocorrer em um dos corpos ou em ambos, é importante, para evitar a perda de polimento das restauraçóes e conseqüente acúmulo de biofilme, alteração de cor e volume (Tanaue et al. ${ }^{26}$ 2000). Na Odontologia ela deveria ocorrer de maneira fisiológica, como resultante do desgaste causado pelo contato entre as superfícies dentais (Kawai e Heinfelder ${ }^{15}$ 1995), porém condições patológicas poderiam acelerá-la (Condon e Ferracane ${ }^{7}$ 1996, Craig e Power ${ }^{9}$ 1976).

$\mathrm{O}$ desgaste pode ser classificado em erosão, que são perdas por efeitos químicos; atrição, que são desgastes ocorridos nas superfícies de contato oclusal, e abrasáo, que são desgastes nas superfícies dentais que não se enquadram nas duas primeiras situaçōes. Um exemplo desta última situaçâo são os desgastes sofridos nas superfícies das restauraçóes e dos dentes (oclusal, vestibular e lingual) através da escovação (Mair ${ }^{18}$ 1992; Mair et al. ${ }^{19}$ 1996).

As resinas à base de polimetilmetacrilato têm sido amplamente utilizadas para a confecção das restauraçôes temporárias. Porém, esse material possui algumas características negativas, uma delas é a reação exotérmica intensa que pode chegar a $82^{\circ} \mathrm{C}\left(\right.$ Vallitu $\left.^{27} 1996\right)$, tornando necessário o procedimento de remoção e inserção da restauração durante toda a polimerização, e assim resultando numa discrepância marginal da restauração (Moulding et al. ${ }^{21}$ 1994). Outro fator negativo é a discrepância marginal resultante da contração de polimerização (Robinson e Hojivitra ${ }^{24}$ 1992), e a presença de monômeros livres nessas resinas, que podem gerar agressão ao tecido pulpar (Grajower et al. ${ }^{11} 1979$ ).

As resinas à base de bysacril para confecção de restauraçóes temporárias possuem como característica fa- vorável uma reação exotérmica de 29,50 C à 35,60 C (Lieu et al. ${ }^{17}$ 2001), não sendo necessária toda a série de cuidados durante a polimerização (não agressão à polpa) (Christensen ${ }^{5}$ 1996). Outras características relevantes desse material seriam a moderada estabilidade da cor (Scotti et al..$^{25}$ 1997); e a apresentaçâo de um sistema de auto-mistura, o que levaria a uma proporção correta e a uma economia do material (Young et al. ${ }^{28}$ 2001). Como inconveniente essas resinas à base de bysacril apresentam menor resistência a fratura em relação àquelas de polimetilmetacrilato (Osman e Owen ${ }^{23}$ 1993).

Como as resinas para confecção de coroas temporárias desgastam-se com a utilização, é necessário verificar a resistência ao desgaste em três resinas após a simulação de um ano de escovação, uma à base de polimetilmetacrilato (Duralay - Reliance Dental) e duas à base de bysacril (InstaTemp Provisional Crown \& Bridge - Sterngold ImplaMed; Perfect Temp - Discus Dental).

\section{MATERIAL E MÉTODO}

Três matrizes foram usinadas em aço inoxidável com $5 \mathrm{~mm}$ de diâmetro por $3 \mathrm{~mm}$ de altura para a obtenção dos corpos-de-prova.

Três resinas para confecção de coroas temporárias foram selecionadas: InstaTemp Provisional Crown \& Bridge - Sterngold ImplaMed, Perfect Temp - Discus Dental e Duralay - Reliance Dental.

Em temperatura ambiente de aproximadamente $21^{\circ} \mathrm{C}$ com umidade relativa em torno de $50 \%$, doze corpos-de-prova $(n=12)$ de cada material restaurador foram obtidos seguindo-se as especificações de cada fabricante.

Os trinta e seis corpos-de-prova foram armazenados em água destilada à $37^{\circ} \mathrm{C}$ durante catorze dias (Condon e Ferracane ${ }^{7}$ 1996; Heath e Wilson ${ }^{12}$ 1976). Decorrido esse período, todos os corpos-de-prova foram pesados em uma balança analítica (Mettler Toledo, modelo AB204) no Centro de Pesquisa e Tecnologia da Johnson \& Jonhnson ${ }^{\circledR}$ - São José dos Campos.

Uma base de aço inoxidável foi usinada, contendo três orifícios padronizados, nos quais foram assentados os corpos-de-prova das diferentes marcas comerciais, de forma que apenas $1 \mathrm{~mm}$ do corpo-de-prova ficou saliente em relaçáo à superfície da base de aço inoxidável. Três corpos-de-prova de cada grupo eram testados simultaneamente a cada 6 horas, o que resultou em um total de 24 horas de simulação para cada grupo.

A base contendo os corpos-de-prova foi adaptada e 
Nishioka RS, Mesquita AMM, Kojima AN, Duarte Filho G, Vasconcelos LG. Avaliação da resistência ao desgaste de três resinas para coroas temporárias após simulação de um ano de escovação. Revista de Odontologia da Universidade Cidade de São Paulo 2008 janabr; $20(1): 46-51$

presa a uma cuba também de aço inoxidável, pertencente ao corpo do aparelho simulador de escovação "Smile" do Centro de Pesquisa e Tecnologia da Johnson \& Jonhnson ${ }^{\circledR}$ - São José dos Campos, que executou 80 ciclos lineares por minuto. Essa cuba serviu também para conter uma solução de $6 \mathrm{ml}$ de água para $6 \mathrm{ml} \mathrm{de}$ dentifrício (Sorriso - Colgate, com abrasivo principal: carbonato de cálcio).

Escovas de textura média TEK® (Johnson \& Jonhnson), foram adaptadas a um dispositivo do aparelho simulador que realizava os movimentos lineares, com amplitude suficiente que atingia os três corpos-de-prova num único movimento, sendo que durante todo o processo de simulação foi utilizada uma carga axial programada no próprio aparelho de $200 \mathrm{~g}$ ((Deboer et al. ${ }^{10}$ 1985) de força.

A substituição das escovas ocorreu a cada 90 minutos, o que correspondeu a 3 meses de simulação. Após 360 minutos de ensaio, que corresponderam a um ano de escovação (Aboprev ${ }^{1}$ 1997), os corpos-de-prova foram pesados novamente na mesma balança analítica, com o objetivo exclusivo de se observar as variações de peso resultantes do desgaste da escovação.

Para se compararem os valores médios de cada grupo individualmente antes e após a escovação, foi efetuado o teste $t$ (Student). Realizou-se também Análise de Variância (um fator) e para a comparação entre os grupos foi realizado o teste de Tukey. O nível de significância adotado foi o valor convencional de 5\%.

\section{RESULTADOS}

A média e desvio-padrão dos dados obtidos em gra-
Tabela 3: Comparação entre as médias de perda de massa dos grupos $(g)$

\begin{tabular}{ccc}
\hline \hline Grupos & $\begin{array}{c}\text { Médias de perda } \\
\text { de massa (d.p) }\end{array}$ & Conjunto \\
\hline Duralay & $0,0017(0,0004)$ & $\mathrm{A}$ \\
Perfect Temp & $0,0013(0,0005)$ & $\mathrm{A}$ \\
Insta Temp & $0,0003(0,0007)$ & $\mathrm{B}$ \\
\hline \hline
\end{tabular}

Letras iguais representam médias estatisticamente semelhantes. ${ }^{*} \mathrm{p}<0.05$

mas na pesagem é apresentada na Tabela1.

O teste $t$ (Student) quando aplicado, para cada material restaurador, possibilitou rejeitar a hipótese de igualdade entre o desgaste médio antes e após a escovação. Assim, para o Duralay, obteve-se uma diminuição de peso $(0,1023-0,1006=0,0017)$ estatisticamente significante $(\mathrm{t}=14,56 ; \mathrm{gl}=11 ; \mathrm{p}=0,001)$; enquanto para o Perfect Temp, obteve-se uma diminuição de peso $(0,0864-0,0851=0,0013)$ estatisticamente significante $(\mathrm{t}=8,95 ; \mathrm{gl}=11 ; \mathrm{p}=0,001)$, e, finalmente, para o Insta Temp, obteve-se uma diminuição de peso $(0,1061$ $-0,1058=0,0003)$ não estatisticamente significante $(\mathrm{t}$ $=1,66 ; \mathrm{gl}=11 ; \mathrm{p}=0,124)$.

Os dados obtidos para os valores de massa foram analisados pelo teste de análise de variância de uma entrada considerando o fator de variância o tipo de resina (Tabela 2) e o teste de Tukey, apresentado na Tabela 3, foi utilizado para a comparação entre grupos. Observou-se que houve diferença estatisticamente significante $(\mathrm{p}<0,05)$ entre os tipos de resina. A resina Duralay teve os valores mais altos de perda de massa, enquanto Insta Temp apre-

Tabela 1. Média e desvio padrão dos dados obtidos na pesagem (g), antes e após a escovação, segundo o material restaurador.

\begin{tabular}{cccccccccc}
\hline \hline \multirow{2}{*}{ Estatística } & \multicolumn{3}{c}{ DURALAY } & \multicolumn{3}{c}{ PERFECT TEMP } & \multicolumn{3}{c}{ INSTA TEMP } \\
\cline { 2 - 10 } & Antes & Depois & Diferença & Antes & Depois & Diferença & Antes & Depois & Diferença \\
\hline N & & 12 & & & 12 & & & 12 & \\
Média & 0,1023 & 0,1006 & 0,0017 & 0,0864 & 0,0851 & 0,0013 & 0,1061 & 0,1058 & 0,0003 \\
DP & 0,0019 & 0,0020 & 0,0004 & 0,0021 & 0,0020 & 0,0005 & 0,0028 & 0,0031 & 0,0007 \\
\hline \hline
\end{tabular}

Tabela 2: Resultados da Análise de variância: tipo de resina e tipo de resina $X$ quantidade de desgaste

\begin{tabular}{cccccc}
\hline \hline $\begin{array}{c}\text { Fonte de } \\
\text { Variância }\end{array}$ & $\begin{array}{c}\text { Graus de } \\
\text { Liberdade }\end{array}$ & $\begin{array}{c}\text { Soma de } \\
\text { Quadrados }\end{array}$ & $\begin{array}{c}\text { Quadrado } \\
\text { Médio }\end{array}$ & F & P \\
\hline Tipo de resina & 2 & $8.672 \mathrm{E}-06$ & $4.336 \mathrm{E}-06$ & 18.1 & $0.00001^{*}$ \\
Resina X desgaste & 33 & $7.896 \mathrm{E}-06$ & $2.393 \mathrm{E}-07$ & & \\
\hline \hline
\end{tabular}


Nishioka RS, Mesquita AMM, Kojima AN, Duarte Filho G, Vasconcelos LG. Avaliação da resistência ao desgaste de três resinas para coroas temporárias após simulação de um ano de escovação. Revista de Odontologia da Universidade Cidade de São Paulo 2008 janabr; 20(1):46-51

sentou os valores mais baixos.

\section{DISCUSSÃO}

O desenvolvimento de novas tecnologias trouxe algumas opções de resinas ao mercado para a confecção de restauraçōes temporárias, e a característica principal destas é a elevada resistência ao desgaste. O processo de desgaste depende de uma série de fatores que determinam a magnitude do desgaste como a duração, a localização, as superfícies envolvidas, os efeitos químicos (Mair et al. ${ }^{19}$ 1996), o abrasivo (Andrade et al. ${ }^{2}$ 1998), a dureza das cerdas da escova (Consani $e t a l .^{8}$ 1995), a força e a velocidade da escovaçáo. Outros fatores são o tipo e concentração de carga da resina, reação de polimerização, união da carga à matriz, propriedades físicas, reação química, e condições de carga mecânica (Kernet et al. ${ }^{16}$ 1999).

A lisura superficial é um fator determinante na qualidade do material restaurador (Bargui e Simmons ${ }^{3}$ 1976; Chung $\left.^{6} 1994\right)$, que deveria ser resistente ao desgaste provocado pela escovação para evitar o acúmulo do biofilme que traria risco de cárie e doença periodontal influenciando negativamente na manutenção estética (Tanoue et al. ${ }^{26} 2000$, Heath e Wilson ${ }^{12}$ 1976).

Baseando-se no protocolo de trabalhos de Condon e Ferracane $^{7} 1996$, Heath e Wilson ${ }^{12} 1976$, os corpos-deprova permaneceram em água destilada durante catorze dias a $37^{\circ} \mathrm{C}$, para se aproximarem das condiçóes do meio bucal. Quando é medida a perda do material por alteração de peso, um controle sobre a água é fundamental, caso contrário a desidratação e reidratação podem levar a um erro de cálculo da perda real de peso causada pela abrasão (Momoi et al. ${ }^{20}$ 1997). A utilização de um perfilômetro seria interessante, como teste complementar, ajudando na verificação de formação de irregularidades e média de material removido (Grajower et al. ${ }^{11} 1979$; Andrade Jr et al. ${ }^{2}$ 1998).

O simulador de escovação "Smile" executou 80 ciclos lineares por minuto, o que corresponderia a quatro sessões de escovação ao dia, uma vez que cada área de escovação deveria ter em média 20 ciclos. A substituição das escovas ocorreu a cada 90 minutos o que correspondeu à simulação de três meses de escovação. Visualmente a substituição foi caracterizada pela falta de manutenção da padronização linear dos tufos, marcada pela divergência das cerdas.

Obedecendo à mesma conduta laboratorial utilizada por De Boer et al. ${ }^{10}$ (1985), durante todo o processo foi registrada no aparelho de simulação uma carga axial de $200 \mathrm{~g}$, que teoricamente simulou a pressão ideal que o paciente deveria aplicar durante o procedimento de escovação.

Este trabalho utilizou uma cuba de aço inoxidável contendo uma de soluçáo de $6 \mathrm{ml}$ de água para $6 \mathrm{~g}$ de dentifrício (Consani et al. ${ }^{8}$ 1995) como auxiliar no desgaste superficial das amostras. Uma das propriedades terapêuticas dos dentifrícios é a sua pequena capacidade abrasiva, e essa propriedade teve uma incidência sobre a superfície dentária e sobre o material restaurador (Heath e Wilson ${ }^{12}$ 1976; Johannsen et al. ${ }^{13}$ 1989).

Os resultados deste trabalho demonstraram que não houve diferença estatisticamente significante entre a resina Duralay e a Perfect Temp, enquanto que a Insta Temp obteve perda de massa significativamente menor.

Uma comparação direta com outros trabalhos foi difícil, pois não há uma padronização nas metodologias. Concorda-se com Momoi et al. ${ }^{20}$ (1997) que também constatou que há ausência de trabalhos nessa linha de pesquisa com esses materiais para se estabelecer uma comparaçáo de resultados.

Os testes de abrasão realizados no laboratório não refletiram as condições orais normais, pois não foi simulado o desgaste provocado por contato de dente natural, nem pelos diferentes materiais restauradores e muito menos por diferentes tipos de alimentação (Kao $\left.{ }^{14} 1989\right)$. Sendo assim, os resultados apresentados demonstraram simplesmente o desgaste por abrasão provocado por escova dental.

\section{CONCLUSÃO}

Todas as resinas testadas tiveram alteração em seu peso, portanto as três (Duralay, Perfect Temp e Insta Temp) são susceptíveis ao desgaste por escovação. A resina Insta Temp foi a mais resistente ao desgaste gerado pela escovação, pois foi a que menos perdeu peso comparativamente à Perfect Temp e Duralay.

\section{AGRADECIMENTOS}

Ao Centro de Pesquisa e Tecnologia da Jonhson \& Jonhson do Brasil que possibilitou a realização deste trabalho, ressalta-se o conhecimento e colaboração dos cientistas Arnaldo Antonio Ditlef, Paulo César de Godoy, Luiz B. Simionato. 


\section{REFERÊNCIAS}

1. Aboprev. Promoção de Saúde Bucal. Rio de Janeiro. Rio de Janeiro: Artes Médicas, 1997 475p.

2. Andrade Junior ACC, Andrade MRTC, Machado WAS, Fischer RG. Estudo in vitro da abrasividade de dentifrícios. Rev Odontol Univ São Paulo 1998 Jul; 12(3):231-6.

3. Bargui N, Simmons JR EW. The marginal integrity of the temporary acrylic resin crown. J Prosthet Dent 1976 Sep; 36(3): 274-7.

4. Carranza, F.A. Periodontia Clínica de Glickman, $7^{\mathrm{a}}$ ed. Rio de Janeiro: Guanabara Koogan, 1992 754p.

5. Christensen GJ. Provisional restorations for fixed prosthodontics. J Am Dent Assoc 1996 Feb; 127(2): 249-52.

6. Chung K. Effects of finishing and polishing procedures on the surface texture of resin composites. Dent Mater 1994 Sep; 10(5): 325-30.

7. Condon JR, Ferracane JL. Evaluation of composite wear with a new multi-mode oral wear simulator. Dent Mater 1996 Jul; 12(4): 218-26.

8. Consani S, De Goes MF, Sinhoreti MAC, Sobrino LC. Avaliação in vitro da abrasão produzida por dentifrícios fluoretados comerciais. Semina 1995; 16(2): 308-12.

9. Craig RG, Powers J M. Wear of dental tissues and materials. Int Dent J 1976 Jul; 26(2): 121-33.

10. De Boer P, Duinkerke ASH, Arends J. Influence of toothpaste particle size and tooth brush stiffness on dentine abrasion in vitro. Caries Res 1985; 19(3):232-9.

11. Grajower R, Sharaboshi S, Kaufman E. Temperature rise in pulp chamber during fabrication of temporary self-curing resin crowns. J Prosthet Dent 1979 May; 41(5): 535-40.

12. Heath JR, Wilson HJ. Abrasion of restorative materials by toothpaste. J Oral Rehabil 1976 Apr; 3(2): 121-38.
13. Johannsen $G$, Redmalm G, Ryden H. Surface changes on dental materials. The influence of two different dentifrices on surface roughness measured by laser reflexion and profilometer techniques. Swed Dent J 1989; 13(6)267-76.

14. Kao EC. Influence of food simulation solvents on resin composites and glassionomer restorative cement. Dent Mater 1989 May; 5(3): 201-8

15. Kawai K, Leinfelder KF. In vitro evaluations of OCA wear resistance of posterior composites. Dent Mater 1995 Jul; 11(4):246-51.

16. Kern M, Strub JR, Lü XY. Wear of composite resin veneering materials in a dual axis chewing simulator. J Oral Rehabil 1999 May; 26(5):372-8.

17. Lieu C., Nquyen TM, Pavant L. In vitro comparison of peak polymerization temperatures of 5 provisional restoration resins. J Can Dent Assoc 2001 Jan; 67(1): 36-9.

18. Mair LH. Wear in dentistry: current terminology. $J$ Dent 1992 Jun; 20(3): 140-4.

19. Mair LH, Stolarskita TA, Vowlis RW, Lloyd CH. Wear mechanisms, manifestations and measurement: report of a workshop. J Dent 1996 Jan-Mar; 24(1-2):141-8.

20. Momoi Y, Hirosaki K, Kohno A, Mc Cabe JF. In vitro toothbrush-dentifrice abrasion of resin-modified glass ionomers. Dent Mater 1997 Mar; 13(2):82-8.

21. Moulding MB, Loney RW, Ritsco RG. Marginal accuracy of provisional restorations fabricated by different techniques. Int J Prosthodont 1994 Sep-Oct; 7(5): 468-72.

22. Nishioka RS, Bottino MA. Próteses provisórias em prótese parcial fixa. Rev Inst Ciênc Saúde 1989; 7(2):114.

23. Osman YI, Owen CP. Flexural strength of provisional restorative materials. J Prosthet Dent 1993 Jul; 70(1): 94-6.

24. Robinson FB, Hojivitra S. Marginal fit of direct temporay crowns. J Prosthet Dent 1982 Apr; 47(4): 390-2. 
Nishioka RS, Mesquita AMM, Kojima AN, Duarte Filho G, Vasconcelos LG. Avaliação da resistência ao desgaste de três resinas para coroas temporárias após simulação de um ano de escovação. Revista de Odontologia da Universidade Cidade de São Paulo 2008 janabr; 20(1):46-51

25. Scotti R, Mascelloni SC, Fortini F. The in vitro color stability of acrylic resins for provisional restorations. Int J Prosthodont 1997 Mar-Apr; 10(2): 164-8.

26. Tanoue N, Matsumura H, Atsuta M. Wear and surface roughness of current prosthetic composites after toothbrush/dentifrice abrasion. J Prosthet Dent 2000 Jul; 84(1): 93-7.
27. Vallitu P. K. Peak temperatures of some prosthetic acrylates on polymerization. J Oral Rehabil 1996 Nov; 23(11): 776-81.

28. Yonug HM, Smith CT, Morton D. Comparative in vitro evaluation of two provisional restorative materials. J Prosthet Dent 2001 Feb; 85(2): 129-32.

Recebido em: 16/10/2006 Aceito em: 18/10/2007 